upstairs were better results in adductor group. IV morphine consumption within the first 48 hours period were less in adductor group comparing to femoral group.

Conclusions Adductor nerve block showed better early recovery in revision TKA when comparing to femoral nerve block (FNB).

\section{INTERMEDIATE CERVICAL PLEXUS BLOCK FOR INTERNAL JUGULAR VEIN CATHETERIZATION IN ONCOLOGY PATIENTS. A PROSPECTIVE, RANDOMIZED STUDY}

${ }^{1} \mathrm{~F}$ Sifaki ${ }^{*},{ }^{1} \mathrm{~S}$ Bagntasarian, ${ }^{1} \mathrm{G}$ Pistiolas, ${ }^{1} \mathrm{P}$ Christidis, ${ }^{2} \mathrm{E}$ Liolios, ${ }^{1} \mathrm{E}$ Koraki. ${ }^{1}$ Georgios Papanikolaou, General Hospital of Thessaloniki, Thessaloniki, Greece; 'Lewisham and Greenwich NHS Trust, London, UK

\subsection{6/rapm-2021-ESRA.195}

Background and Aims Internal Jugular Vein Catheterization (IJVC) in oncology patients can be challenging, as most of these patients have undergone multiple painful procedures and fear of pain. This study aims to determine whether intermediate cervical plexus block (ICPB) is superior to conventional local anesthesia in terms of satisfaction of the patient for IJVC.

Methods 30 oncology patients, ASA III, were randomly divided into two groups. In Group A $(n=18)$, ICPB with lidocaine $2 \%(5 \mathrm{ml})$ was administered to the patients, 5 minutes before the placement of the central venous catheter (CVC). In Group B $(n=12)$, conventional local anesthesia with lidocaine $2 \%(5 \mathrm{ml})$ was administered at the site of the catheterization, 5 minutes before the placement of CVC. The procedure in both groups was ultrasound-guided. The duration, complications and VAS score of the procedure along with patient's satisfaction of the procedure were recorded. Patient's vital signs were recorded throughout the procedure.

Results No complications were reported in both Groups. The mean duration of the procedure was not statistically significant between the two groups. VAS scores and Satisfaction Scores were found to be statistically significant between the two groups.

Conclusions In this study, the performance of ICPB in oncology patients undergoing IJVC was found to provide superior analgesia and comfort to the patients when compared to conventional local anesthesia. To the authors' concern there are no studies exploring the superiority of ICPB as a means of analgesia to patients undergoing IJVC. Certainly, more studies should be performed for further confirmation of these findings.

\section{INNOVATIVE AND HI-FIDELITY SIMULATOR FOR FASCIAL PLANE BLOCKS}

${ }^{1} \mathrm{P}$ Fusco*, ${ }^{2} \mathrm{GM}$ Petroni, ${ }^{2} \mathrm{~S}$ Tullij, ${ }^{2} \mathrm{~V}$ Arcangeli, ${ }^{2} \mathrm{G}$ Balestra, ${ }^{2} \mathrm{~F}$ Marinangeli. ${ }^{1}$ Ospedale San Salvatore, L'Aquila, Italy; ${ }^{2}$ University of L'Aquila, L'Aquila, Italy

\subsection{6/rapm-2021-ESRA.196}

Background and Aims Ultrasound guided regional anesthesia (UGRA) is an invasive procedure that involves complex motor skills. ${ }^{1}$ As ultrasound-guided regional anesthesia (UGRA) represents the gold standard for performing regional blocks, there is a need for learning the technical skills associated with this technique. $^{2,3}$

Methods We developed a innovative and high-fidelity system simulator for ultrasound-guided fascia blocks based on real ultrasound images, named Block Sim ${ }^{\mathrm{TM}}$, made by Accurate.

Results Block Sim allows us to practice on: anterior thoracic wall blocks [PECS I, PECS II, serratus plane block and parasternal block (figure 1)], paraneuraxial nerve blocks (Paravertebral block and erector spine plane block (ESP), and abdominal wall blocks (transversus abdominis plane block (TAP), quadratus lumborum block (QLB I e II). Thanks to the ultrasound probe software, this new simulation system allows the physician to view real ultrasound images. Users can move the ultrasound probe in different directions on the available insert and observe a coherent real-time modification of the scene. Users can also perform a simulated injection of the local anesthetic, using an external syringe, when the tip of the needle is inside of the fascia.The user can practice and develop competency using simulation scenarios based on real clinical images and, at the end of the procedure, an indicative global score will be given to the user based on his performance.

Conclusions We believe that the use of simulators is very important, first of all for patient safety, and for anesthetists who can finally make mistakes to learn.
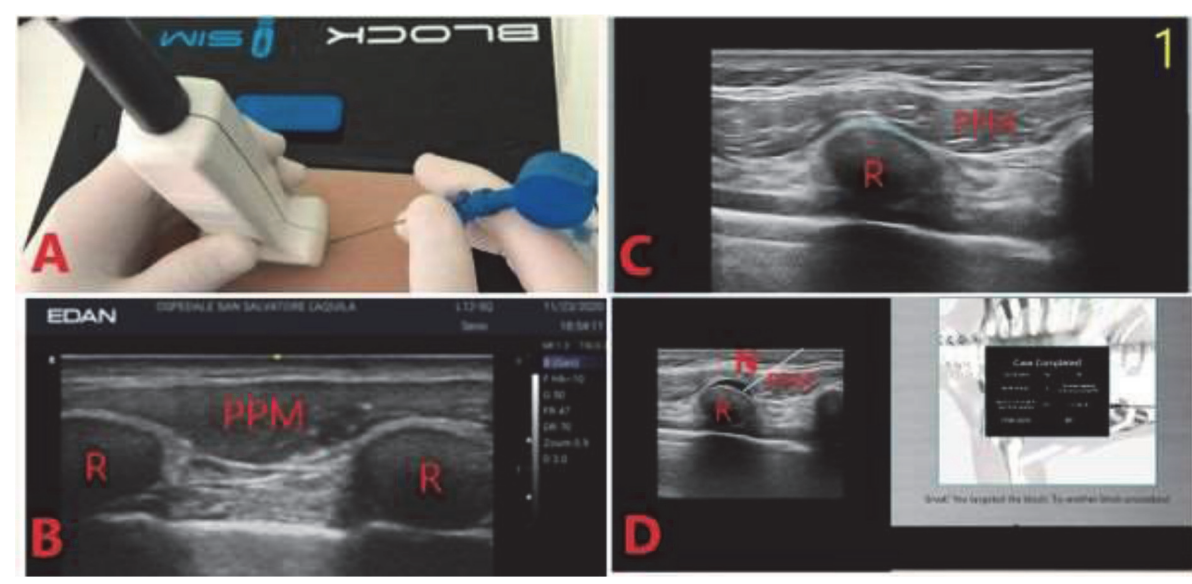

Abstract 196 Figure 1 B real Ultrasound images C,D BlockSim's images 